\title{
Stronger Tests of the Collapse Locality Loophole in Bell Experiments
}

\author{
Adrian Kent* \\ Centre for Quantum Information and Foundations, DAMTP, \\ Centre for Mathematical Sciences, University of Cambridge, \\ Wilberforce Road, Cambridge, CB3 OWA, United Kingdom and \\ Perimeter Institute for Theoretical Physics, 31 Caroline Street North, Waterloo, ON N2L 2Y5, Canada.
}

\begin{abstract}
Several versions of quantum theory assume some form of localized collapse. If measurement outcomes are indeed defined by localized collapses, then a loophole-free demonstration of Bell nonlocality needs to ensure space-like separated collapses associated with the measurements of the entangled systems. This collapse locality loophole remains largely untested, with one significant exception probing Diosi's and Penrose's gravitationally induced collapse hypotheses. I distinguish two versions of the loophole, and focus on the more strongly motivated version, in which local hidden variables may depend causally on collapse outcomes but may not independently depend on measurement settings. I describe here techniques that allow much stronger experimental tests. These apply to all the well known types of collapse postulate, including gravitationally induced collapse, spontaneous localization models and Wigner's consciousness-induced collapse.
\end{abstract}

\section{INTRODUCTION}

Bell's work [1, 2] on the empirical implications of the Einstein-Podolsky-Rosen argument [3] showed that quantum theory violates local causality [4]. It has led to many beautiful experiments that aim to refute local hidden variable theories (LHVT). To eliminate as many extra assumptions as possible, and so refute as wide a class of LHVT as possible, experiments have been devised that close or minimize the detector efficiency [5] 8 ] and locality and settingindependence loopholes 9 12. Analysis of the memory loophole showed that it too can be closed by using appropriate statistical tests in standard Bell experiments [13, 14.

More recently, what were described "loophole-free" experiments, closing all three loopholes simultaneously, were announced [15-17. While these were undoubtedly impressive experiments that achieved a long-sought goal, their definitiveness was overstated. Significant and theoretically interesting loopholes remain. In particular, the collapse locality loophole [18 remains largely untested. This loophole arises because, while Bell experiments are supposed to demonstrate non-local correlations between measurement outcomes on spacelike separated systems, we do not know for sure where in spacetime the relevant measurement outcomes actually arise. In some versions of quantum theory this question does not have a well-defined answer. However, in versions in which collapse is an objective and localized process, it does. There is not a consensus among theorists that objective collapse hypotheses with localized collapses are necessary, and they may not be correct. However, there are a variety of good motivations for taking them seriously, given the problems in making sense of unitary quantum theory [19] and quantum gravity [20. It is questionable whether any Bell experiment to date has created spacelike separated measurement outcomes, according to most well-known collapse hypotheses (e.g. 2123]).

For example, the Ghirardi-Rimini-Weber dynamical collapse model 22 requires a measurement-type interaction to create a superposition of distinguishable position states for a large number of correlated particles in order to produce a significant probability of a definite measurement event. Similarly, mass-dependent continuous spontaneous localization models 23 25] require the creation of a superposition of significantly different mass distributions in order to produce a significant probability of a definite measurement event. The photodetector avalanches generated by photon measurements in many Bell experiments do not involve sufficiently many particles, and the states of wires carrying currents transmitting the results are not sufficiently distinguishable, according to the criteria these models define. Of course, dynamical collapse models do predict that collapses defining measurement outcomes eventually take place, but we expect this to be later in the causal chain, when the results are correlated and stored in computer memory, printed out, or read by experimenters. There is no guarantee that such collapses are spacelike separated. Indeed, only one Bell experiment to date [26] has been specifically designed to ensure that, given a specific objective collapse hypothesis (due to Penrose and Diosi), spacelike separated collapses define the measurement outcomes in the two wings. We consider this experiment, and its relation to the collapse hypotheses of Refs. [22 25], in more detail below.

Tests of the collapse locality loophole can be motivated as tests of standard quantum theory against a class of apparently internally consistent, albeit strange, alternatives, collectively termed causal quantum theory [18, 27. However, the loophole per se does not logically rely on the consistency or plausibility of causal quantum theory. Testing it tests quantum theory against the general class of local hidden variable theories in which collapses, and 
thus measurement outcomes, causally influence and may be causally influenced by the local hidden variables. We will assume this more general motivation here. We are interested in testing the hypothesis that spacelike separated measurement outcomes respect Bell inequalities. This is consistent with the outcomes of pairs of measurements that are simultaneous and co-located, or lightlike or timelike separated, respecting standard quantum predictions (and thus violating Bell inequalities). We need to assume this in order to explain the results of Bell experiments to date.

Ideally, one would ultimately like to close as many loopholes as possible in a single experiment: hence the motivation for experiments such as those in Refs. [15 17. However, this involves tradeoffs, and it may not yet be possible to close all plausible loopholes simultaneously. For this reason, many pre-2015 Bell experiments were designed to close only a single loophole, or subset of loopholes. One motivation for addressing loopholes separately is the intuition that we should assign a relatively small Bayesian prior to the hypothesis that nature exploits any given unclosed loophole, and a much smaller prior probability to the hypothesis that nature exploits two or more loopholes simultaneously in a way that is only evident when both or all apply. Put another way, while hidden variable theories that exploit one loophole might just possibly have some theoretical motivation, those that require two or more in combination seem far more conspiratorial and implausible.

This justification seems particularly strong in the case of the collapse locality loophole. As noted above, there are theoretical motivations for considering the hypothesis that localized collapses play a fundamental role in physics, and if what we call measurements are fundamentally defined by localized collapses then we really need to close the collapse locality loophole in order to demonstrate Bell non-local correlations between measurement outcomes. It is not immediately obvious that this line of thought adds any particular motivation to hidden variable theories that might exploit other loopholes. This motivates us to consider experiments that test the collapse locality loophole as strongly as possible given current technology, without considering other loopholes, and we focus on such experiments in this paper.

In the standard framework for describing Bell experiments, the collapse locality loophole can be understood as follows. In a schematic description of a standard Bell experiment (Fig. 1) a source S generates entangled particles which propagate to devices in wings $\mathrm{L}$ and $\mathrm{R}$. Measurement setting choices $A$ and $B$ in the two wings are made locally, producing outcomes $a$ and $b$ respectively. A local hidden variable theory would allow these outcomes to depend on a common local hidden variable $\lambda$, which depends on events at the source $\mathrm{S}$ and in its past light cone. In principle, the local hidden variables at L may depend on other events in its past light cone (and similarly R). We make here the common assumption that the relevant events in the past light cone of $\mathrm{L}$ (including those in the past light cone of S) are effectively uncorrelated with the measurement choice B, and similarly $\mathrm{R}$ and A, excluding superdeterminist explanations for Bell correlations. To simplify the notation we list explicitly only the dependences crucial for our discussion; thus we write $\lambda=\lambda(S)$ to emphasize its dependence on events at and in the past of S.

Now consider the possibility that the outcome of measurement A is actually determined by a collapse event at a point $\mathrm{C}$ in the causal future of the collapse event determining the outcome of measurement $B$ (Fig. 2). In this case the local hidden variables at $\mathrm{C}$ may depend explicitly on this prior collapse event, and hence on the choice $B$ and outcome $b$ as well as on events at $\mathrm{S}$.

We need to distinguish here between two hypotheses associated with collapse and locality in Bell experiments. Since these have not previously been clearly separated, we propose to refine the terminology.

In principle, the local hidden variables at $\mathrm{C}$ could depend on everything in their past light cone. In particular, they could depend on the measurement choices $B$, simply because a configuration has been chosen for the measurement apparatus at $\mathrm{R}$ and this physical fact per se may influence variables in the future light cone in a general local hidden variable theory. An explanation of this type effectively relies on the locality loophole, together with the hypothesis that the relevant measurement event actually takes place at a point $(\mathrm{C})$ timelike separated from the point $(\mathrm{R})$ where the measurement settings were made, even though the experiment was intended to make measurements at spacelike separated points ( $\mathrm{L}$ and $\mathrm{R}$ ). We refer to this as exploiting the extended collapse locality loophole.

A more restrictive hypothesis is that the local hidden variables depend significantly on the choice $B$ and outcome $b$ only if a collapse event indeed took place at R. On this view, simply setting up the measurement apparatus at R so as to define a particular choice of measurement does not per se have a relevant effect - an effect that significantly alters the predicted correlations - on the local hidden variables at $\mathrm{C}$, even though $\mathrm{C}$ is in the future light cone of $\mathrm{R}$. If the measurement at $\mathrm{R}$ does not produce a collapse event there, then the local hidden variables at $\mathrm{C}$ may be treated as though effectively independent of the measurement settings. However, a collapse of the subsystem at $\mathrm{R}$ is a physical event that may affect the local hidden variables of the entangled subsystem at $\mathrm{C}$, in such a way as to alter the predicted correlations. According to quantum theory, a collapse at $\mathrm{R}$ with outcome $b$ projects the state of the local subsystem onto the eigenstate with eigenvalue $b$ of the observable defined by measurement choice $B$, a state which depends on both $B$ and $b$. The hypothesis is that such collapse events significantly affect the local hidden variable dynamics, so that the local hidden variables in the causal future of $\mathrm{R}$ may depend on both $B$ and $b$ in a way 


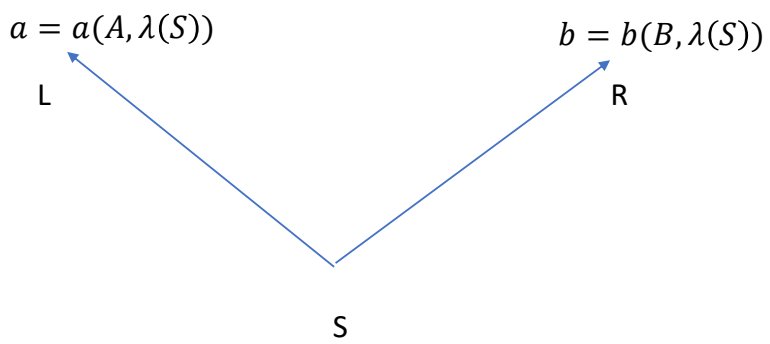

FIG. 1: Schematic description of a standard Bell experiment

that affects the observed correlations. In particular, since $\mathrm{C}$ is in the future light cone of $\mathrm{R}$, the local hidden variables there may depend on both $B$ and $b$. We refer to this as exploiting the essential collapse locality loophole.

Our motivation for this second hypothesis, and for the term "essential", comes from applying EPR's [3] and Bell's [1, 2, 4 arguments to models where collapses are physically objective and localized. EPR's discussion of measurements on entangled systems suggested that quantum theory may be incomplete, since - given premises that are arguable, although of course presently generally rejected - a measurement on one subsystem seems to change the physical properties of a distant subsystem. Bell's discussion of locally causal hidden variables suggests a natural way in which more complete underlying theories, consistent with Einstein causality, could be defined. Theories with objective localized collapses offer a precise definition of measurement, and hence of the events to which the EPR argument should apply. They also introduce localized physical events (the collapses) and locally created physical data (the collapsed states) that supplement and modify unitary quantum dynamics. A local hidden variable theory underlying an objective localized collapse version of quantum theory thus ought to allow the distribution of local hidden variables for a system to depend causally on collapse data associated with that system as well as on its initial quantum state.

Another possibility (Fig. 3) is that the outcomes of measurements $A$ and $B$, apparently obtained at L and $\mathrm{R}$, are actually jointly determined by co-located collapse events at $\mathrm{C}$. In this case the local hidden variables at $\mathrm{C}$ determining both outcomes ( $a$ and $b$ ) may depend explicitly on both measurement settings (A and B). This hypothesis may be justified if the detectors at $\mathrm{L}$ and $\mathrm{R}$ are similar, and the detector readings are transmitted along similar channels to a device at $\mathrm{C}$ that stores them and calculates correlations. With this set up, an objective localized collapse theory will generally either predict collapses in the vicinity of both $\mathrm{L}$ and $\mathrm{R}$ (either in the detectors or at an early stage within the communication channels) or neither. If neither, then it may predict no collapse until the quantum state recording the outcome data undergoes appropriate further evolution and/or interactions. The qualitative and quantitative features of the evolution and interactions required to induce collapses are determined by the specifics of the collapse theory. The essential and extended versions of the collapse locality loophole are hard to separate in this case, since the localized collapse theory may imply that there is effectively a single joint measurement of $A$ and $B$ at $\mathrm{C}$.

In either of the configurations described in Figs. 22 and 3, local hidden variable theories can reproduce the predictions of quantum theory, violating Bell inequalities and producing "faux non-local" correlations, which appear to verify Bell non-locality but actually do not.

Discussion of the collapse locality loophole [18] motivated a beautiful experiment by Salart et al.[26], which tested 


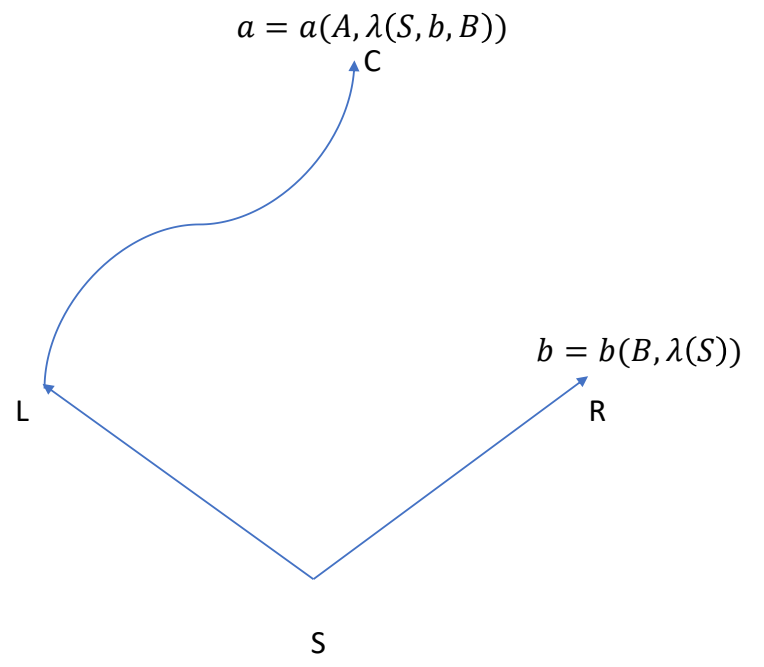

FIG. 2: A Bell experiment in which the collapse locality loophole allows records of outcomes, apparently from one wing of the experiment but actually generated at a point in the causal future of both, to depend on measurement settings and outcomes in the other wing. If the outcomes are indeed generated by collapse events at $\mathrm{R}$, then both versions of the collapse locality loophole allow the outcomes at $\mathrm{C}$ to depend on the settings and outcomes at $\mathrm{R}$.

the loophole for the specific collapse hypotheses sketched by Diosi [28] and Penrose [29], according to which wave function collapse takes place to prevent a superposition of distinguishable gravitational fields. Diosi and Penrose proposed quantitative estimates for the distinguishability of mass distributions in superposition components required for collapse, and Salart et al. were able to arrange a configuration of piezocrystals coupled to a Bell experiment so that, on Diosi and Penrose's estimates, the relevant collapses would indeed be spacelike separated.

The Salart et al. experiments confirmed the predictions of quantum theory, thus closing the loophole under the precise assumptions made. That said, Diosi and Penrose's estimates were based on heuristic calculations rather than derived from a consistent underlying theory, and altering them by a factor of $\approx 10^{2}$ would leave the loophole open in the Salart et al. experiment. The experimental analysis did not address other hypotheses about gravitationally induced collapse, or other types of spontaneous collapse models (e.g. 22, 23). Nor, of course, did the experiment address Wigner's speculative (but motivated) hypothesis 21] that measurement outcomes and collapse might require conscious observation.

There thus remains very strong motivation for stronger, more systematic and more general tests of both forms of the collapse locality loophole. In this paper I describe simple techniques that enable some tests of the essential collapse locality loophole.

\section{Empirical tests: Bell experiments}

Non-local correlations appear to have been demonstrated in many Bell experiments. However, because of the collapse locality loophole, appearances may possibly have been deceptive. Consider again a typical Bell experiment involving an entangled pair of photons, generated by a source S, whose polarizations are measured by a combination of filters and photodetectors in local labs at space-like separated points L and R, following the layout of Fig. 3.

Speaking loosely - in the way that physicists do when discussing quantum experiments when conceptual details are not relevant - a photon entering, say, lab L enters a photodetector, generates an avalanche of photons, which generates a small electrical signal. Similar events take place in lab R. The signals are brought together and compared, 


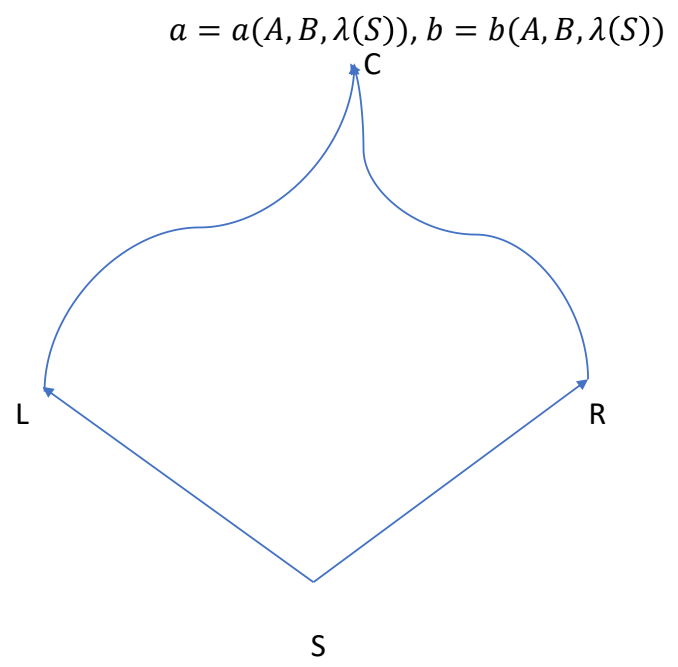

FIG. 3: Another application of the collapse locality loophole. Here the records of outcomes, apparently from both wings, are generated together at point $\mathrm{C}$. The recorded measurement outcomes for each wing may depend on both measurement settings.

producing a record of the results, and allowing their correlations to be obtained, at some point $\mathrm{C}$ in the joint future of $\mathrm{L}$ and $\mathrm{R}$.

The point at issue, in the context of the collapse locality loophole, is where and when physical measurement(s) and collapse(s) actually take(s) place in such experiments. Is amplification of a single photon state to a larger number of photons is sufficient to define a measurement? Or generating a small electrical pulse from the photodetector? According to most well-known collapse hypotheses, the answers are no, or at least not necessarily. The relevant collapses and measurement outcomes may instead have been co-located, at or after C. For example, they may take place when the measurement outcomes were amplified and recorded on a hard drive, or when the printout was read by an experimenter.

In the experiment mentioned above, Salart et al. 26] sent the electrical signals generated by the photomultipliers directly through piezocrystals on each wing, causing the piezocrystals to deform. These deformations were observed locally by interferometry, using mirrors attached to one end of each piezocrystal. The deformation of the piezocrystals and consequent movement of the mirrors involves a relatively large mass displacement, far larger than that created by the photomultiplier avalanche or the electrical pulses. The experimental parameters were chosen so that, according to Diosi and Penrose's intutions and estimates, the two possible states of the piezocrystal (deformed and undeformed) create macroscopically distinct gravitational fields, which quickly causes a collapse when the experiment would (according to unitary quantum dynamics) place them in superposition.

Salart et al.'s experiment verifed that the piezoactuator displaced a $2 \mathrm{mg}$ mirror of dimensions $3 \times 2 \times 0.15 \mathrm{~mm}$ through a distance of $\geq 12.6 \mathrm{~nm}$ within $\approx 6 \mu \mathrm{s}$ of the photon entering the analyzer. In collapse models, until collapse takes place, measurement processes such as those in the experiment produce superposition states. The relevant superposition here is of the undisplaced and displaced states. Salart et al. obtain a lower bound on the collapse time of such a superposition by neglecting the actuator displacement and considering the state of the mirror, which is effectively in a superposition of two overlapping position states. This gives [30] a collapse time of $\leq \approx 1 \mu \mathrm{s}$, using Diosi's estimate (Penrose's estimate is a factor of two smaller), and hence an upper bound of $\approx 7 \mu$ s between the photon entering the analyzer and a collapse event.

The two wings in the Salart et al. experiment were separated by $\approx 60 \mu \mathrm{s} \times c$, so that under the stated assumptions the introduction of the piezocrystals ensures spacelike separated collapses in the two wings. Without the piezocrystals, 
according to Diosi-Penrose, there would be no space-like separated collapses, since the mass displacements due to the photomultiplier avalanches and electrical pulses are negligible over the experimental timescales.

Salart et al. thus successfully closed the essential collapse locality loophole for gravitationally induced collapse, assuming that Diosi-Penrose's collapse time estimates are accurate. It should be noted, though, that these estimates are based on intuition and require some rather ad hoc assumptions, and that they are not known to follow from a consistent dynamical theory of gravitationally induced collapse. Somewhat lower collapse time bounds could be derived by allowing for the difference in densities between the piezoactuator and mirror and modelling the mass distribution of both. Still, it is unclear that the experiment would close the loophole given an extra factor of $10^{2}$ or so in the Diosi-Penrose estimates. This already gives motivation for seeking stronger experiments.

\section{Other collapse models}

It is interesting to consider the collapse times predicted in the Salart et al. experiments by other well known collapse models. The most extensively studied, and arguably the best motivated, of these is the mass-dependent version of the continuous spontaneous localization model due to Ghirardi, Pearle and Rimini [23 25]. We make the same simplifying assumption as Salart et al., by considering only the state of the mirror, which before collapse is in a superposition of overlapping position states. An analysis of the collapse rate [35] gives a collapse time of

$$
\frac{1}{4 \pi \lambda a^{2}} N^{-2} A,
$$

where $\lambda$ and $a$ are the free parameters in the CSL model, $N$ is the number of nucleons in the sliver of the mirror that does not overlap both superposition states, and $A$ is the area of the mirror surface. For the parameter choices often used in example discussions, $\lambda=10^{-16} \mathrm{~s}^{-1}, a=10^{-5} \mathrm{~cm}$, this gives a collapse time of $\approx 10^{-8} \mathrm{~s}$, faster than the Diosi-Penrose estimate.

However, it is suggested in the literature [31 33 that a value of $\lambda$ as low as $10^{-19} \mathrm{~s}^{-1}$ suffices to produce collapses fast enough to be consistent with human perceptions of definite events, which are the only certain data from which lower bounds can be derived. There are significant uncertainties in the derivation of this estimate, which may be too low to ensure that photon observations generally cause collapses within the human eye (see e.g. 34]). On the other hand, one can argue [32, 34 that observers generally produce physiological responses to any significant observation, and that a collapse model may need only to ensure that collapses take place very rapidly after such a response. If this is accepted, values of $\lambda$ significantly lower than $10^{-19}$ may be consistent with our perceptions. Indeed, if the relevant physiological responses are essentially always macroscopic - say, involving at least $1 \mathrm{~g}$ of body matter moving at least $1 \mathrm{~mm}$ - then a firm lower bound would be many orders of magnitude lower. It is thus hard to argue that the Salart et al. experiment has definitely closed the CSL version of the essential collapse locality loophole.

Similar comments apply to the earlier Ghirardi-Rimini-Weber (GRW) collapse model. This model, in its original version, requires us to treat all the nucleons in the relevant system as distinguishable particles, each of which has the same collapse rate. It is thus natural to consider the piezocrystal and mirror as one system. We can simplify the estimate by taking the two superposition states to be displacements of the entire system by the average value $\approx \frac{d}{2}$ (in the actual states, the nucleon displacements range from 0 to $d$ ). This gives a GRW collapse time estimate of

$$
\frac{16 a^{2}}{\lambda N d^{2}}
$$

where $\lambda$ and $a$ are GRW model paramters, $d$ is the displacement and $N$ the number of nucleons in the piezocrystal and mirror. For the often used example values $\lambda=10^{-16} \mathrm{~s}^{-1}$ and $a=10^{-5} \mathrm{~cm}$, this gives a collapse time of $\approx 2 \times 10^{-4} \mathrm{~s}$. The Salart et al. experiment thus did not close the GRW version of the essential collapse locality loophole even with these standard example parameters. Finding a firmly defensible lower bound for the parameter $\lambda$ is as problematic for GRW models as for CSL models, so that the Salart et al. experiment is far from closing the GRW version of the essential collapse locality loophole.

\section{Extended experiments}

We can schematically summarize a class of experiments to test the collapse locality loophole, including that of Salart et al., by Fig. 4. A standard Bell experiment takes place, with a source $S$ and detectors $D_{L}$ and $D_{R}$ whose measurement settings are adjustable: they are adjusted by $A$ and $B$ (or by their appropriately programmed devices) to 
choose random or pseudo-random settings for each run. The measurement outcomes for each run are propagated via channels $C_{L}$ and $C_{R}$. They are then amplified by apparatus $A_{L}$ and $A_{R}$, with the amplification processes taking place in space-like separated regions $R_{L}$ and $R_{R}$. The full process on each wing $X$, from entering the detector $D_{X}$ to the completion of amplification by $A_{x}$, takes place in a region which we denote by $R_{X}^{\prime}$. In the Salart et al. experiments, the regions $R_{L}^{\prime}$ and $R_{R}^{\prime}$ were also space-like separated.

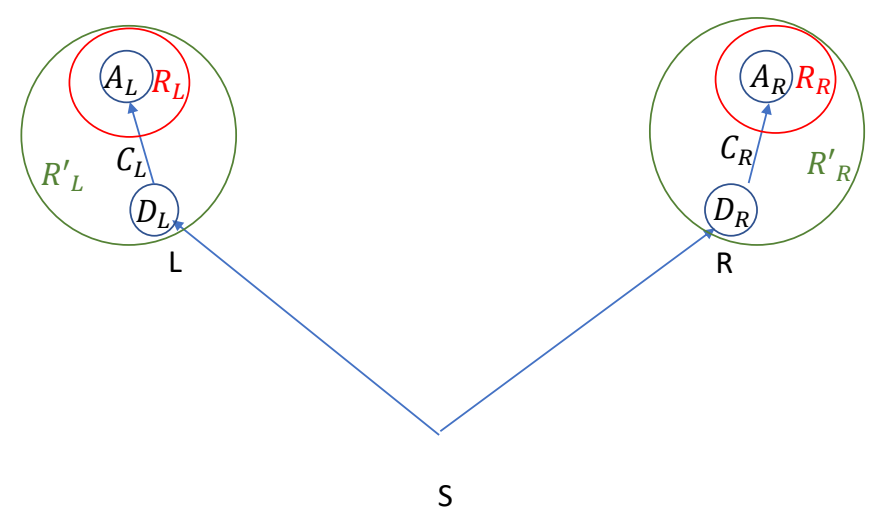

FIG. 4: Schematic description of an experiment designed to close some version of the collapse locality loophole. The detector readings are communicated via channels to apparatus which, in an appropriate sense, amplify them to ensure that collapses are induced. The regions $R_{L}$ and $R_{R}$ are space-like separated.

Any given such experiment is designed to test the loophole given the hypothesis that collapses take place because of amplification by the apparatus $A_{L}$ and $A_{R}$ and within the regions $R_{L}$ and $R_{R}$. For an interesting experiment, this hypothesis must be based on well motivated theoretical ideas, which must also imply that collapse does not typically take place either in the detectors $D_{X}$ (where $X=L$ or $R$ ) or the channels $C_{X}$. If it did, then the apparatus would be unnecessary. Moreover, in this case the experiment might or might not be testing the loophole, depending whether the regions during which the collapses typically take place are spacelike separated. In particular, if the regions $R_{L}^{\prime}$ and $R_{R}^{\prime}$ are not space-like separated, then typical pairs of collapses may not necessarily be space-like separated. On the hypothesis that the collapses take place during propagation in the channels, then whether they are space-like separated depends on the collapse duration and the channel configurations. (We do not necessarily assume the channels propagate light-like signals.) On the hypothesis that the collapses take place within the detectors, and the detector measurements take place in space-like separated regions (as in standard Bell experiments), then standard Bell experiments would already have closed the loophole.

At first sight, it may seem that the channels should necessarily be very short. One might also think that the entire regions $R_{L}^{\prime}$ and $R_{R}^{\prime}$, including all the processes from the systems entering the detectors to the completion of the amplification, should necessarily be space-like separated. Both of these conditions were satisfied in the Salart et al. experiment. Indeed, the space-like separation of $R_{L}^{\prime}$ and $R_{R}^{\prime}$ is required in order to close the extended version of the collapse locality loophole, which effectively exploits the locality loophole, so that local hidden variables in the causal future of $D_{X}$ may be significantly influenced by the measurement settings of these detectors. However, neither of the above conditions is required to close the essential collapse locality loophole, in which local hidden variables are significantly influenced only by (and in the causal futures of) collapse events. On the hypothesis that collapses are caused by interaction with $A_{X}$ and take place within the regions $R_{X}$ the detectors $D_{X}$ and channels $C_{X}$ effectively form part of the entangled system measured in an extended Bell experiment. To test the essential collapse locality 
loophole, all that matters is that $R_{L}$ and $R_{R}$ are space-like separated.

This gives us considerable freedom in designing experiments. In particular, the channels may be slow and long, compared to the other experimental parameters. Moreover, the (by hypothesis uncollapsed) detector "measurements" need not even be space-like separated. For example, several interesting versions of the essential collapse locality loophole could be tested by a Bell experiment with nearly adjacent detectors, with outcomes propagated to antipodal points on the Earth by fibre optic links or radio signals, followed by suitable synchronised amplification at the antipodes (see Fig. 5). Stronger tests still could be carried out by experiments in which one or both amplifying devices are located in space (see Fig. 6). Again, the Bell experiment detectors need not be widely separated, and could both be on Earth, so no long range controlled distribution of entangled photons is required.

These experiments have the unusual feature that they are based on the assumption that they generate long range entanglement of subsystems that include some degrees of freedom (such as electrical signals) normally treated as classical. This assumption follows from some specified collapse model or hypothesis. It is not required that the entangled subsystems be precisely identified or isolated: decohering interactions with the environment are not necessarily problematic. However, the experiment needs to ensure that any such decohering interactions are not of the form that, according to the relevant collapse hypothesis, leads to collapse in the past of the apparatus. For example, humans peeking at the detector output data before they arrive at the final apparatus could invalidate the tests of the Wigner version of the collapse locality loophole described below.

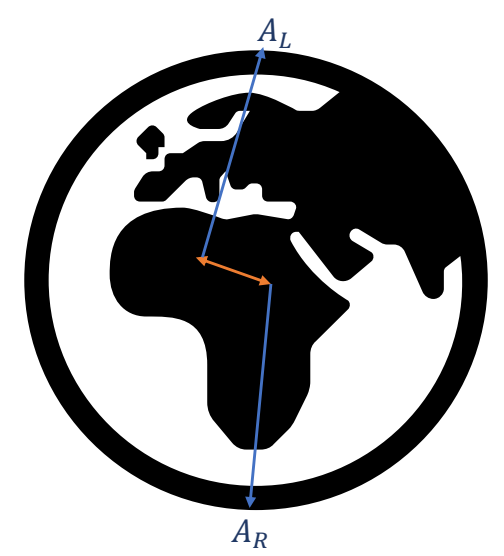

FIG. 5: A long range terrestrial experiment designed to test the essential collapse locality loophole. The detector readings from wings of a short range Bell experiment are communicated to amplifying apparatus at antipodal points. By introducing delays if necessary, they are input into the apparatus nearly simultaneously in rest frame, so as to maximize the collapse time for which space-like separated collapses would ensure.

To illustrate some of the power of this technique, note that the Salart et al. experiment could be modified by adding terrestrial channels to antipodal points, with synchronization of the propagation of the signals through antipodal piezocrystals. This would allow a separation of the Earth's diameter, i.e. $\approx 1.24 \times 10^{4} \mathrm{~km} \approx 40 \mathrm{~ms} \times c$, an improvement by a factor of $\approx 10^{3}$, allowing a corresponding margin against the considerable theoretical uncertainties in the collapse time estimates for Diosi and Penrose's proposals and for other collapse models. The increased time length would also allow scope for larger and slower piezocrystals and for other means of generating distinct mass distributions in response to signals. It is an interesting challenge for technological ingenuity to identify the largest scale event that can safely be created, conditioned on a particular signal, within $\approx 40 \mathrm{~ms}$. As well as piezocrystals, such an event could involve motors, triggered springs, and/or controlled explosions, for example. Space-based experiments give even 


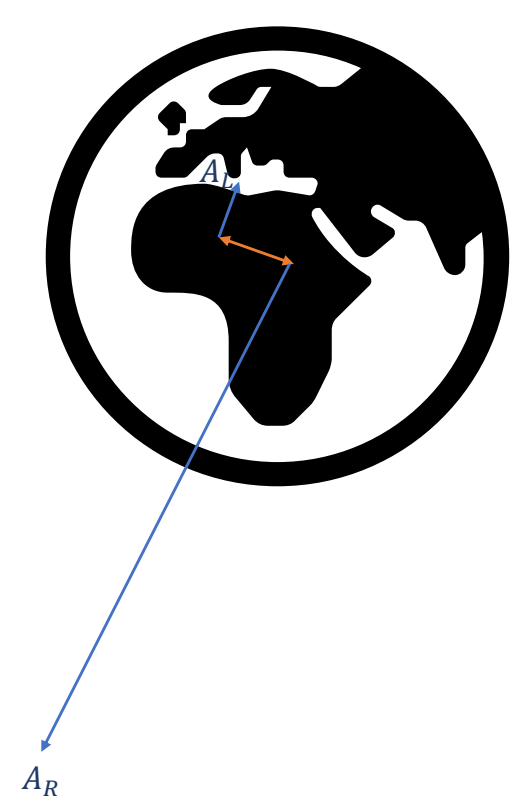

FIG. 6: A partly space-based version of the previous experiment. One signal is sent to an apparatus on a space-based laboratory, while the other goes to an apparatus on a ground station. To test the Wigner version of the essential collapse locality loophole, the apparatus may include human observers.

longer time intervals and correspondingly more scope (though for large separations perhaps less critical need) for ingenuity.

A test of the essential collapse locality loophole based on Wigner's hypothesis is also relatively practical with this technique, requiring only one astronaut who need carry no specialized equipment. Typical human perception or reaction times of $\approx 100-200 \mathrm{~ms}$ require only that one human participant is at least $\approx 2-5 \times 10^{4} \mathrm{~km}$ from Earth, assuming that the other is on the opposite side of the Earth; a medium Earth orbit falls within this range.

Wigner's hypothesis, of course, proposes a direct link between consciousness and objective collapse. It is generally disfavoured, because (a) many physicists think that consciousness is weakly emergent from (i.e. in principle entirely explicable by) known physics, (b) many physicists think that quantum physics is very plausibly complete, i.e. that there is no quantum measurement problem and no need to add anything to the quantum description of reality, (c) even among those who believe there is a hard problem of consciousness and a quantum reality problem, most find it hard to see how Wigner's hypothesis can fit into a plausibly attractive underlying theory. Still, many might agree that these are very deep questions, that our best present theories may yet need radical revision, our best present intuitions are not necessarily a very good guide, and experiments would be worth carrying out for those reasons alone.

One can also motivate these experiments via dynamical collapse models. We have already noted that lower bounds on the mass-dependent CSL and GRW collapse model parameters can only be justified by analysing human conscious perceptions. This also applies to other collapse models that could be considered alongside CSL and GRW. Ultimately, the only firm reason for believing that a well motivated collapse model must define a definite measurement event is that humans would definitely be conscious of the measurement outcome if they observed it. The simplest (and perhaps only) way of ensuring this must be the case for all potentially interesting collapse models is to directly involve a human observer in the tests. This gives quite a strong motivation for tests of the essential collapse locality loophole directly involving human observers, independent of Wigner's hypothesis. 


\section{CONCLUSIONS}

Collapse hypotheses can be motivated as solutions to the quantum reality (or measurement) problem, as alternative routes to unifying quantum theory and gravity without necessarily quantising gravity in any standard sense, or even as speculative ways of connnecting consciousness and physics. All of these motivations (which may also be combined in various ways) are questionable, but all have thoughtful proponents. If collapses are objective, it is quite plausible that they are typically well localized events, and indeed this is a feature of some explicit collapse models.

Bell non-locality is not necessarily connected with or problematic for any of these motivations, and it seems pretty likely that it is a fundamental fact about nature; this is certainly the straightforward explanation of Bell experiments to date. However the problems of quantum theory, the difficulty in unifying quantum theory and gravity and the mystery of consciousness all counsel a little humility: it is still possible that we understand nature much less well than we imagine. And a demonstrable failure of Bell nonlocality (despite appearances) would radically alter the theoretical landscape, particularly in connecting quantum theory and gravity. All of this motivates testing Bell nonlocality as thoroughly as possible, particularly since Bell experiments are also interesting technological and experimental challenges, with spin-offs in applied physics, and relatively inexpensive.

Causal quantum theory [18, 27] is an explicit alternative to quantum theory that exploits the essential collapse locality loophole. However, the loophole may be exploited in other ways. Tests that could refute causal quantum theory [27] (based on some specific localized collapse hypothesis) thus would not necessarily close the essential collapse locality loophole (based on the same hypothesis).

There is also a cryptographic motivation for considering Bell experiment loopholes and how to close them. It is often crucial for future users of quantum cryptography and quantum communication systems to guard against eavesdropping or cheating by testing that states involving allegedly entangled separated subsystems genuinely are entangled states of the correct form. In principle, Bell experiments are certifications of entanglement. However, for users working with untrusted devices, in principle, every unclosed Bell experiment loophole gives adversaries a cheating strategy. In particular, the collapse locality loophole focuses attention on whether users know for sure when and where their Bell measurement outcomes are actually generated.

For all these reasons, we hope and expect that our techniques will be exploited and extended. We have focussed on experiments aimed at closing the essential collapse locality loophole, assuming that nature does not exploit collapse locality in combination with other loopholes. This follows in the tradition of many significant Bell experiments that addressed either the detector efficiency or the locality loophole but not both. Nonetheless, although we are not aware of any interesting alternative to quantum theory that exploits the extended collapse locality loophole, or other combinations of loopholes including collapse locality, we cannot exclude the possibility that one might be devised. It would thus also be desirable to design further, even more definitive, experiments that could ultimately simultaneously close the locality, collapse locality, detector efficiency and other loopholes.

I am very grateful to Philip Pearle for many helpful suggestions and for estimates of collapse rates in the CSL and GRW models.

This work was partially supported by FQXi and by Perimeter Institute for Theoretical Physics. Research at Perimeter Institute is supported by the Government of Canada through Industry Canada and by the Province of Ontario through the Ministry of Research and Innovation.

* Electronic address: A.P.A.Kent@damtp.cam.ac.uk

[1] John S Bell. On the problem of hidden variables in quantum mechanics. Rev. Mod. Phys., 38:447, 1966.

[2] John Stuart Bell. On the Einstein-Podolsky-Rosen paradox. Physics, 1:195-200, 1964. Reprinted in John Stuart Bell, Speakable and Unspeakable in Quantum Mechanics, Cambridge University Press, 1987.

[3] Albert Einstein, Boris Podolsky, and Nathan Rosen. Can quantum-mechanical description of physical reality be considered complete? Physical Review, 47(10):777, 1935.

[4] John S Bell. The theory of local beables. Epistemological Letters, 9(11), 1976.

[5] P. M. Pearle. Hidden-variable example based upon data rejection. Phys. Rev. D., 2:1418, 1970. URL http://link.aps. org/doi/10.1103/PhysRevD.2.1418

[6] M. A. Rowe, D. Kielpinski, V. Meyer, C. A. Sackett, W. M. Itano, C. Monroe, and D. J. Wineland. Experimental violation of a Bell's inequality with efficient detection. Nature (London), 409:791, 2001. URL http://www.nature.com/nature/ journal/v409/n6822/full/409791a0.html.

[7] D. N. Matsukevich, P. Maunz, D. L. Moehring, S. Olmschenk, and C. Monroe. Bell inequality violation with two remote atomic qubits. Phys. Rev. Lett., 100:150404, 2008. URL http://link.aps.org/doi/10.1103/PhysRevLett.100.150404 
[8] M. Giustina, A. Mech, S. Ramelow, B. Wittmann, J. Kofler, J. Beyer, A. Lita, B. Calkins, T. Gerrits, S. W. Nam, R. Ursin, and A. Zeilinger. Bell violation using entangled photons without the fair-sampling assumption. Nature (London), 497:227, 2013. doi: 10.1038/nature12012. URL http://www.nature.com/nature/journal/v497/n7448/full/nature12012.html.

[9] A. Aspect, J. Dalibard, and G. Roger. Experimental test of Bell's inequalities using time-varying analyzers. Phys. Rev. Lett., 49:1804, 1982. URL http://link.aps.org/doi/10.1103/PhysRevLett.49.1804.

[10] G. Weihs, T. Jennewein, C. Simon, H. Weinfurter, and A. Zeilinger. Violation of Bell's inequality under strict Einstein locality conditions. Phys. Rev. Lett., 81:5039, 1998. URL http://link.aps.org/doi/10.1103/PhysRevLett.81.5039.

[11] W. Tittel, J. Brendel, H. Zbinden, and N. Gisin. Violation of Bell inequalities by photons more than $10 \mathrm{~km}$ apart. Phys. Rev. Lett., 81:3563, 1998. doi: 10.1103/PhysRevLett.81.3563. URL http://link.aps.org/doi/10.1103/PhysRevLett.81.3563.

[12] N. Gisin and H. Zbinden. Bell inequality and the locality loophole: Active versus passive switches. Phys. Lett. A, 264:103, 1999. doi: 10.1016/S0375-9601(99)00807-5. URL http://dx.doi .org/10.1016/S0375-9601(99)00807-5.

[13] Jonathan Barrett, Daniel Collins, Lucien Hardy, Adrian Kent, and Sandu Popescu. Quantum nonlocality, Bell inequalities, and the memory loophole. Physical Review A, 66(4):042111, 2002.

[14] Richard D Gill and JA Larsson. Accardi contra bell (cum mundi): The impossible coupling. In Marc Moore, Sorana Froda, and Christian Léger, editors, Mathematical Statistics and Applications: Festschrift for Constance van Eeden, volume 42, pages 133-154. IMS Lecture Notes-Monographs, 2003.

[15] Bas Hensen, Hannes Bernien, Anaïs E Dréau, Andreas Reiserer, Norbert Kalb, Machiel S Blok, Just Ruitenberg, Raymond FL Vermeulen, Raymond N Schouten, Carlos Abellán, et al. Loophole-free Bell inequality violation using electron spins separated by 1.3 kilometres. Nature, 526(7575):682, 2015.

[16] Lynden K Shalm, Evan Meyer-Scott, Bradley G Christensen, Peter Bierhorst, Michael A Wayne, Martin J Stevens, Thomas Gerrits, Scott Glancy, Deny R Hamel, Michael S Allman, et al. Strong loophole-free test of local realism. Physical Review Letters, 115(25):250402, 2015.

[17] Marissa Giustina, Marijn AM Versteegh, Sören Wengerowsky, Johannes Handsteiner, Armin Hochrainer, Kevin Phelan, Fabian Steinlechner, Johannes Kofler, Jan-Åke Larsson, Carlos Abellán, et al. Significant-loophole-free test of Bell's theorem with entangled photons. Physical Review Letters, 115(25):250401, 2015.

[18] Adrian Kent. Causal quantum theory and the collapse locality loophole. Physical Review A, 72(1):012107, 2005.

[19] Simon Saunders, Jonathan Barrett, Adrian Kent, and David Wallace. Many Worlds?: Everett, Quantum Theory, E6 Reality. Oxford University Press, 2010.

[20] Steven Weinstein and Dean Rickles. Quantum gravity. In Edward N. Zalta, editor, The Stanford Encyclopedia of Philosophy. Metaphysics Research Lab, Stanford University, summer 2018 edition, 2018.

[21] Eugene P Wigner. Remarks on the mind-body question. In Philosophical Reflections and Syntheses, pages 247-260. Springer, 1995.

[22] Gian Carlo Ghirardi, Alberto Rimini, and Tullio Weber. Unified dynamics for microscopic and macroscopic systems. Physical Review D, 34(2):470, 1986.

[23] Gian Carlo Ghirardi, Philip Pearle, and Alberto Rimini. Markov processes in Hilbert space and continuous spontaneous localization of systems of identical particles. Physical Review A, 42(1):78, 1990.

[24] Philip Pearle. Combining stochastic dynamical state-vector reduction with spontaneous localization. Physical Review A, 39(5):2277, 1989.

[25] Philip Pearle and Euan Squires. Bound state excitation, nucleon decay experiments and models of wave function collapse. Physical Review Letters, 73(1):1, 1994.

[26] D. Salart, A. Baas, J. A. W. van Houwelingen, N. Gisin, and H. Zbinden. Spacelike separation in a Bell test assuming gravitationally induced collapses. Phys. Rev. Lett., 100:220404, 2008. URL http://link.aps.org/doi/10.1103/PhysRevLett. 100.220404

[27] Adrian Kent. Testing causal quantum theory. Proc. R. Soc. A, 474, 2018. URL http://dx.doi.org/10.1098/rspa.2018. 0501

[28] Lajos Diosi. A universal master equation for the gravitational violation of quantum mechanics. Physics Letters A, 120(8): 377-381, 1987.

[29] Roger Penrose. On gravity's role in quantum state reduction. General Relativity and Gravitation, 28(5):581-600, 1996.

[30] Stephen L Adler. Comments on proposed gravitational modifications of schrödinger dynamics and their experimental implications. Journal of Physics A: Mathematical and Theoretical, 40(4):755, 2007.

[31] Franca Aicardi, Antonio Borsellino, Gian Carlo Ghirardi, and Renata Grassi. Dynamical models for state-vector reduction: do they ensure that measurements have outcomes? Foundations of Physics Letters, 4(2):109-128, 1991.

[32] Philip Pearle. Dynamical collapse. In Philip Pearle, Simon Saunders, and Antony Valentini, editors, Forthcoming book (in preparation). Oxford University Press.

[33] Angelo Bassi, D-A Deckert, and Luca Ferialdi. Breaking quantum linearity: Constraints from human perception and cosmological implications. EPL (Europhysics Letters), 92(5):50006, 2010.

[34] Adrian Kent. Perception constraints on mass-dependent spontaneous localization. In Shan Gao, editor, Quantum Mechanics and Consciousness. Oxford University Press, forthcoming (2020).

[35] Relevant approximations and calculations are set out in detail in Pearle's contribution to a forthcoming book [32. I thank Philip Pearle for an advance copy. 